Isentropic Compression in a Strip Line, Numerical Simulations and Comparison with GEPI Shot 268

A. Lefrançois, P.L. 'Eplattenier, M. Burger

February 22, 2006 
This document was prepared as an account of work sponsored by an agency of the United States Government. Neither the United States Government nor the University of California nor any of their employees, makes any warranty, express or implied, or assumes any legal liability or responsibility for the accuracy, completeness, or usefulness of any information, apparatus, product, or process disclosed, or represents that its use would not infringe privately owned rights. Reference herein to any specific commercial product, process, or service by trade name, trademark, manufacturer, or otherwise, does not necessarily constitute or imply its endorsement, recommendation, or favoring by the United States Government or the University of California. The views and opinions of authors expressed herein do not necessarily state or reflect those of the United States Government or the University of California, and shall not be used for advertising or product endorsement purposes.

This work was performed under the auspices of the U.S. Department of Energy by University of California, Lawrence Livermore National Laboratory under Contract W-7405-Eng-48. 


\title{
ISENTROPIC COMPRESSION IN A STRIP LINE, NUMERICAL SIMULATIONS AND COMPARISON WITH GEPI SHOT 268
}

\author{
A.Lefrancois (*) and P.L’Eplattenier (**), M.Burger(**) \\ Energetic Materials Center, Lawrence Livermore National Laboratory, Livermore, \\ CA 94550 - USA \\ ${ }^{(*)}$ Participating guest at LLNL from DGA/Centre d'etudes de Gramat - France \\ (**) Livermore Software Technology Corporation, 7374 Las Positas Road, CA \\ 94551 - USA
}

Isentropic compressions in a strip line geometry are performed on the GEPI facility at Centre d'etudes de Gramat in order to study isentrope, associated Hugoniot and phase changes [1][2].

3D GEPI configuration has been calculated here to test the new beta version of the electromagnetism package coupled with the dynamics in LS-DYNA ${ }^{\circledR}$ and compared with the GEPI experiment number 268.

\subsection{PRESENTATION OF THE SOFTWARE}

An electromagnetism module is being developed in the general-purpose explicit and implicit finite element program LS-DYNA ${ }^{\circledR}$ in order to perform coupled mechanical/thermal/electromagnetism simulations. The main application of this development is magnetic metal forming. This module allows to introduce some source electrical currents into solid conductors, and to compute the associated magnetic field, electric field, as well as induced currents. These fields are computed by solving the Maxwell equations in the Eddy current approximation, which is a very good approximation for the magnetic metal forming like problems. The Maxwell equations are solved using a Finite Element Method (FEM) for the solid conductors coupled with a Boundary Element Method (BEM) for the surrounding air (or vacuum). Both the FEM and the BEM are based on discrete differential forms. These methods are now implemented on solid hexahedral elements and quadrilateral shells, and will soon be extended to tetrahedra, wedges and triangular shells. 
The electromagnetic fields give the magnetic "Lorentz" force which is added to the LS-DYNA ${ }^{\circledR}$ mechanical solver thus generating deformation of the conductors. This motion is in turn taken into account in the lagrangian computation of the electromagnetic fields.

The electromagnetic fields also give a Joule heating term, which is added to the LS-DYNA ${ }^{\circledR}$ thermal solver. The temperature can in turn be taken into account through an equation of state to get the electromagnetic properties of the materials (for example conductivity).

Since the electromagnetism module is fully integrated into LS-DYNA, all the already existing numerous mechanical and thermal capabilities of the code can be used, and in particular:

The extensive element library with both under-integrated and fully-integrated element formulations includes different solid elements, thick shells, different 3- and 4node shells, beams.

All the 130 metallic and non-metallic material models, many of them equipped with failure criteria, such as metals, plastics, visco-elastic, elasto-viscoplastic, strain rate and temperature dependant plasticity (for example Johnson-Cook, Steinberg-Guinan, Zerilli-Armstrong), superplastic forming option, glass, foam, elastomers, rubbers, explosives.

- $\quad$ The many types of equations of state.

- $\quad$ The efficient contact algorithms with over 25 different contact options.

- $\quad$ The coupled thermo-mechanical capabilities.

- $\quad$ The implicit springback capability.

- $\quad$ The adaptive re-meshing capability.

The electromagnetism solver has already been extensively tested on non-moving conductors with simple geometries where analytical solutions are available. It also has been tested against experimental results on moving conductors with very encouraging results. More details about this module can be found in [3].

Experiments such as the ones performed on GEPI with simultaneous measurements of the total current in the load and the Free Surface Velocity (FSV) are very valuable to benchmark the code. 


\subsection{CONFIGURATION OF THE NUMERICAL SIMULATIONS}

A 3D mesh for the strip line load of GEPI shot 268 has been build with the TrueGrid ${ }^{\circledR}$ mesh generation program. It is presented in Figure 1. It is composed of 15537 solid hexahedral elements, with 10 elements through the thickness of the cooper electrode. The mesh has 1325 BEM faces that are used to compute the surface integrals arising in the BEM method. At the time this report is written, the BEM matrices are assembled and stored as fully dense matrices, which limits the size of the meshes which can be handled, especially on smaller individual machines. Other methods are now introduced to avoid this big memory requirement, which should allow using much larger meshes.

The red part represents the copper strip line, and the blue part is a polyethylene block constraining the movement of the edges of the strip line. The top and bottom electrodes of the strip line are connected to the rest of the generator on the left of the figure.

The simulations have been performed with a surface current approximation, i.e. the diffusion of the current through the thickness of the copper was not taken into account (although it would be possible to do so). The other assumptions were no phase change, no plasma, and a constant and uniform conductivity for the copper electrodes. Further studies should take all that into account. For the electrical Equation of State, a Burgess equation of state [4] is available now, and other ones can be introduced.

The experimental current measured with a Rogowski coil was injected in the strip line.

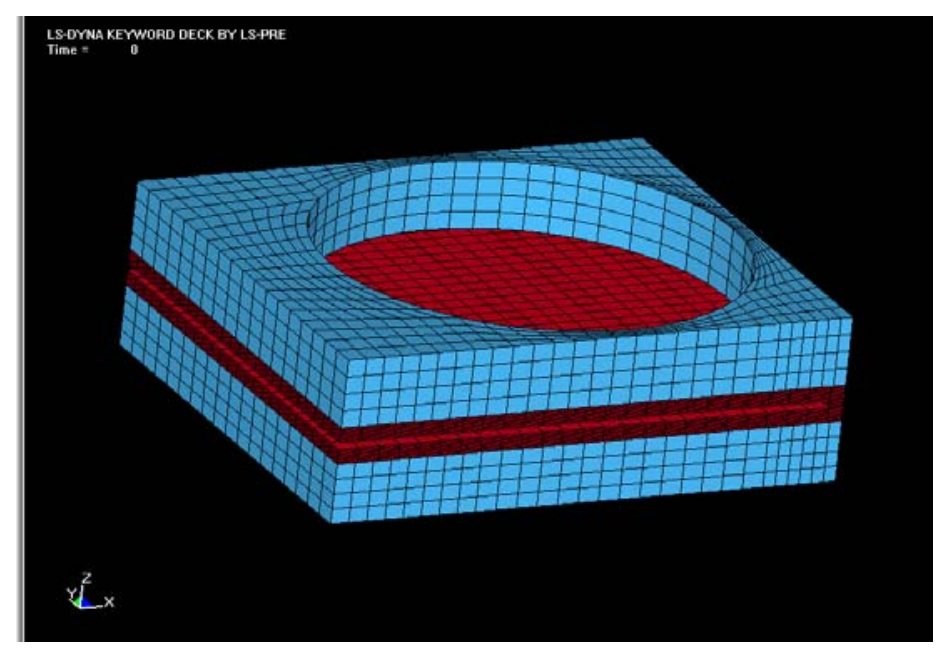

Figure 1 :GEPI strip line configuration 


\subsection{COMPARISONS EXPERIMENTAL-NUMERICAL RESULTS}

A first numerical simulation has been performed using a Zerilli-Armstrong constitutive law for the copper [5].

Figure 2 shows a comparison of between the measured (solid line) and the computed (stars) FSV's for the nominal measured current $(\mathrm{k}=1)$. The numerical FSV is about 24\% higher than the experimental one, but still reproduces the same first 2 oscillations. The phase of the oscillations is lost after the third one, but the period is about the same.

Before doing any further test taking into account the diffusion of the current through the electrodes, the volume Joule heating and a temperature dependant electrical conductivity, a simple test is to check the influence of the input current on the FSV. One has to remember that the experimental current can be measured with a precision of only $10 \%$, and that the Lorentz force varies as the square of the current.

The same simulation was thus performed with only $93 \%$ of the nominal current $(\mathrm{k}=0.93)$. This $7 \%$ decrease in the current induces a large drop in the pressure and thus in the FSV, leading to a numerical FSV very close to the experimental one, as shown on figure 2 .

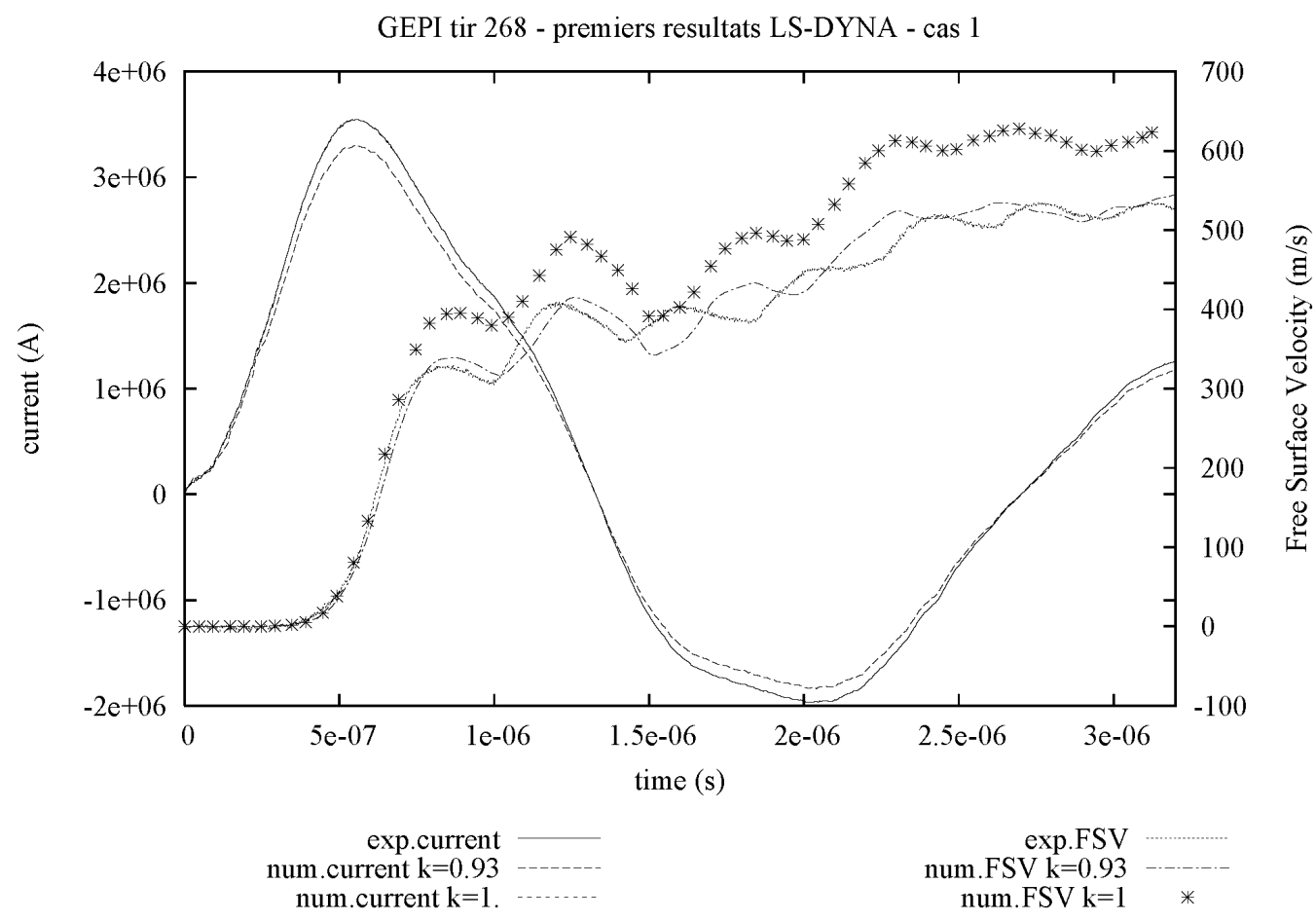

Figure 2 : Numerical currents and FSV for $k=1$ and $k=0.93$ compared to the experimental current and FSV. 
In order to give an idea of the deformation of the electrode during the process, Figure 3 shows the deformed electrode at the end of the simulation (the polyethylene part is not represented).

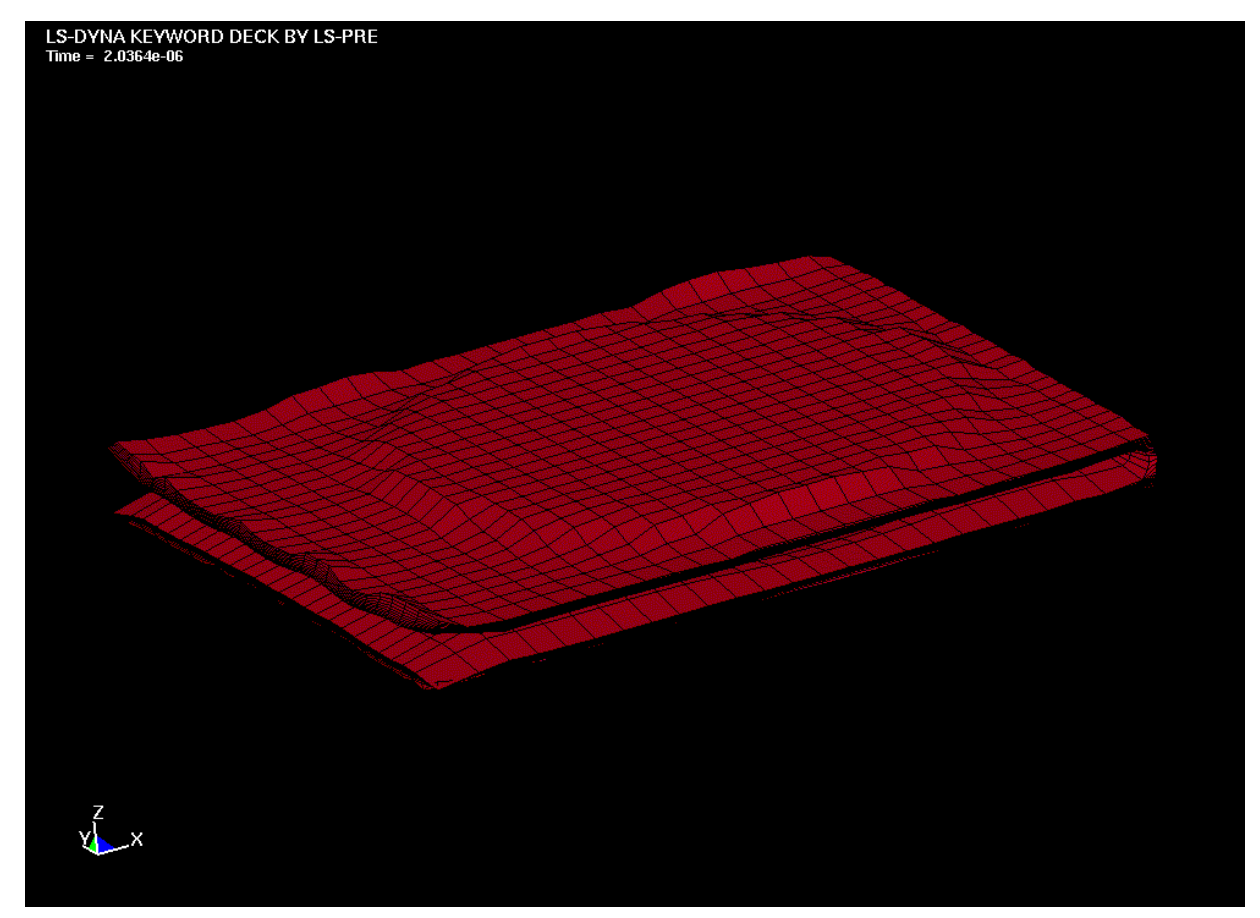

Figure 3: electrode at final time of the simulation

\subsection{INFLUENCE OF THE MATERIAL MODEL}

The influence of different constitutive laws for Copper and the influence of an Aluminum strip line have been investigated. Two constitutive laws (Zerilli-Armstrong, Steinberg) have been compared for the metals [5][6]. Classical Gruneisen equations of state have been applied. The numerical results are presented Figure 4. All of them were done with the nominal experimental current $(\mathrm{k}=1)$.

The Steinberg and Zerilli-Armstrong constitutive laws give the same free surface velocity.

The aluminum strip line with the current and configuration of the copper strip line for shot GEPI 268 has been calculated and give higher free surface velocity. 


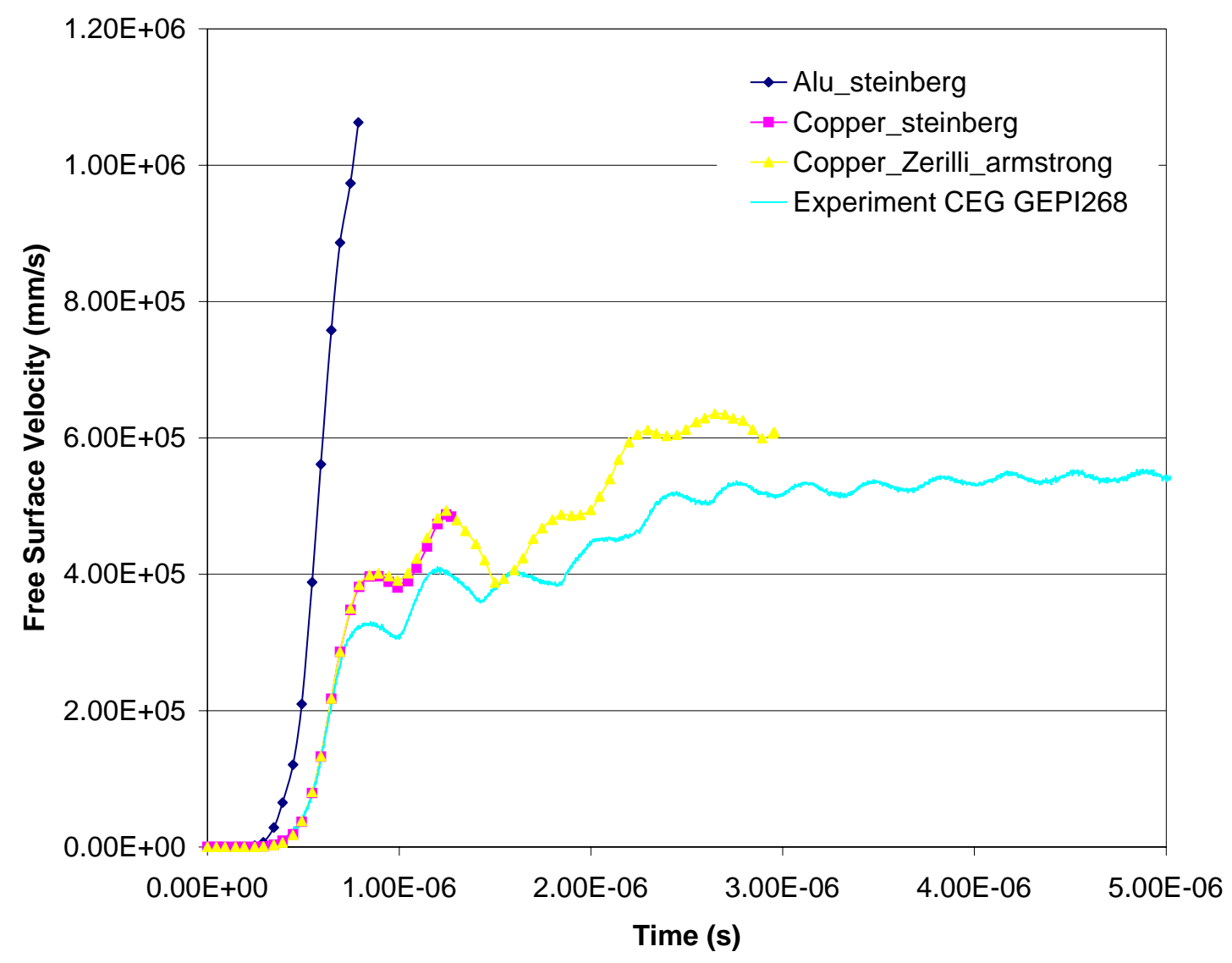

Figure 4 : Free surface velocity versus time, numerical simulations and GEPI shot 268

\subsection{CONCLUSION}

Some further numerical simulations should be performed to test the influence of the different parameters of the simulation, such as :

- The mesh density

- The diffusion of the current through the electrode

- A temperature dependant electrical conductivity

- The material model and equation of state. 
It would be very interesting to benchmark other experimental results on tests performed on GEPI or other generators against LS-DYNA. The relatively simple geometry of strip lines makes them very attractive for such benchmarks.

The code could then be used to understand the complex coupled mechanisms arising in these kinds of problems, such as the diffusion of the current through the thickness of the conductor, and the induced volume repartition of the pressure forces, the influence of the Joule heating on the mechanical and electrical properties of the material, the part of Joule heating and plastic work in the internal energy,...

The code could also be used to optimize other load geometries, using for example the LS-OPT ${ }^{\circledR}$ optimization program.

Another very interesting application of the coupled use of LS-DYNA®, LSOPT ${ }^{\circledR}$ and experimental results would be in the system identification field. The methodology used in LS-OPT consists in an iterative non-linear regression procedure to optimize the parameters of a system or material. This procedure minimizes the errors with respect to given experimental results. The parameters to optimize could for example be the ones appearing in strain rate and temperature dependant constitutive models like the Zerilli-Armstrong [5] or Steinberg [6] ones, for which very little is published. They could also be the ones appearing in the different Equations of State used.

* This Work has been performed under the auspices of the U.S. Department of Energy by the Lawrence Livermore National Laboratory under Contract W-7405-Eng-48

\section{References}

[1] P.L.Hereil, F.Lassale, G.Avrillaud, GEPI : An ICE Generator for Dynamic Material Characterization and Hypervelocity Impact, Shock Compression of Condensed Matter - 2003, pp.1209-1212

[2] P.L.Hereil, Shot GEPI 268 current and free surface velocity data, CEG report 2003

[3] P.L'Eplattenier, "Introduction of an electromagnetism module in LS-DYNA", presented at the Ohio State University workshop on Magnetic Metal Forming, Columbus, OH, May 19, 2005, available at "http://mse-gsd1.eng.ohiostate.edu/ glenn/Mini_Symp/LSTC_Pierrre” 
[4] T.J. Burgess, "Electrical resistivity model of metals", $4^{\text {th }}$ International Conference on Megagauss Magnetic-Field Generation and Related Topics, Santa Fe, NM, USA, 1986

[5] F.J. Zerilli and R.W. Armstrong, "Dislocation-mechanics-based constitutive relations for material dynamics calculations", J.Appl.Phys. 61(5), 1March 1987

[6] D.J.Steinberg, S.G.Cochran, M.W.Guinan, A Constitutive Model for Metals Applicable at High-Strain Rate, J.Appl.Phys.51,1498 (1980) 